\title{
Gynostemma pentaphyllum is neuroprotective in a rat model of cardiopulmonary resuscitation
}

\author{
GERBURG KEILHOFF $^{1}$, TORBEN ESSER ${ }^{2}$, MAXIMILIAN TITZE $^{1}$, UWE EBMEYER $^{2}$ and LORENZ SCHILD ${ }^{3}$ \\ ${ }^{1}$ Institute of Biochemistry and Cell Biology, and Departments of ${ }^{2}$ Anesthesiology and ${ }^{3}$ Pathological Biochemistry, \\ Otto-von-Guericke University Magdeburg, Leipziger, D-39120 Magdeburg, Germany
}

Received June 10, 2017; Accepted October 2, 2017

DOI: $10.3892 /$ etm.2017.5315

\begin{abstract}
Asphyxial cardiac arrest (ACA)-induced ischemia results in acute and delayed neuronal cell death. The early reperfusion phase is critical for the outcome. Intervention strategies directed to this period are promising to reduce ACA/resuscitation-dependent impairments. This study focused on the evaluation of the protective potential of an extract from Gynostemma pentaphyllum (GP), a plant used in traditional medicine with antioxidative, glucose lowering and neuroprotective activities, in an ACA rat model. We tested the following parameters: i) Basic systemic parameters such as $\mathrm{pCO}_{2}$ and blood glucose value within the first $30 \mathrm{~min}$ post-ACA; ii) mitochondrial response by determining activities of citrate synthase, respiratory chain complexes I + III and II + III, and the composition of cardiolipin 6 and 24 h post-ACA; iii) neuronal vitality of the CA1 hippocampal regionby immunohistochemistry $24 \mathrm{~h}$ and 7 days post-ACA; and iv) cognitive function by a novel object recognition test 7 days post-ACA. GP, administered after reaching spontaneous circulation, counteracted the following: i) ACA-mediated increases in arterial $\mathrm{CO}_{2}$ tension and blood glucose values; ii) transient increase in the activity of the respiratory chain complexes II + III; iii) elevation in cardiolipin
\end{abstract}

Correspondence to: Professor Gerburg Keilhoff, Institute of Biochemistry and Cell Biology, Otto-von-Guericke University Magdeburg, Leipziger Strasse 44, Haus 1, D-39120 Magdeburg, Germany

E-mail: gerburg.keilhoff@med.ovgu.de

Abbreviations: ACA, asphyxial cardiac arrest; CA, cornu ammonis; CL, cardiolipin; complex I + III, NADH:cytochrome c oxidoreductase; complex II + III, succinate:cytochrome c oxidoreductase; DMSO, dimethyl sulfoxide; ECG, electrocardiogram; GFAP, glial fibrillary acidic protein; GP, Gynostemma pentaphyllum; IBA1, ionized calcium binding adaptor molecule 1; IPPV, intermittent positive pressure ventilation; MAP, mean arterial pressure; MAP2, microtubule-associated protein 2; NeuN, neuronal nuclei antibody; PBS, phosphate-buffered saline; PFA, phosphate-buffered paraformaldehyde; ROSC, return of spontaneous circulation

Key words: asphyxial cardiac arrest, cardiolipin, dimethyl sulfoxide, hippocampus, mitochondria, object recognition content; iv) hippocampal CA1 neurodegeneration, and v) loss of normal novelty-object seeking. The protective effects of GP were accompanied by side effects of the vehicle DMSO, such as the stimulation of citrate synthase activity in control animals, inhibition of cardiolipin synthesis in ACA animals and complex II + III activity in both control and ACA animals. The results emphasize the importance of the early post-resuscitation phase for the neurological outcome after ACA/resuscitation, and demonstrated the power of GP substitution as neuroprotective intervention. Moreover, the results underline the need of a careful handling of the popular vehicle DMSO.

\section{Introduction}

Cardiac arrest (CA) is a common cause of disability and mortality and thus an important risk to human health. CA initiates an ischemia/reperfusion scenario in the entire organism with particular impact for the CNS. Oxidative stress, excitotoxicity and neuro-inflammation contribute to the impairment of the brain. Within the ischemic phase the deprivation of substrate and oxygen causes dramatic restriction in mitochondrial ATP production which essentially contributes to energetic deficits in the brain. The duration of ischemia determines the extent of damage. Reperfusion results in a dramatic increase in the concentration of reactive oxygen species (ROS). Oxidation of mitochondrial constituents can cause membrane permeabilization and coupled to restriction in ATP production $(1,2)$. As a consequence, cell death may occur.

It has been shown that the early reperfusion phase is of particular impact for the damage of neuronal cells. Therefore, intervention concepts directed to the early phase of cerebral reperfusion are promising for the reduction of early and late impairments of neuronal cells after resuscitation $(3,4)$. Numerous efforts have been undertaken including hypothermia (5), application of antioxidants (3) or noble gases (6) and normoxic resuscitation (7) to improve the neurological outcome in patients after CA. Although significant progress could be achieved there is still a need for further improvement of the intervention including the development of new drugs. According to the AHA's Heart and Stroke Statistics (2017 update) (8), the annual incidence of out-of-hospital CAs in the U.S. is $\sim 111 / 100,000$. Of those patients treated by emergency medical services, only 
$23.8 \%$ survived. This motivated us to study the effect of an extract from Gynostemma pentaphyllum (GP) Makino on CA-depending brain injury. GP is also known as Jiaogulan or xiancao, the 'Herb of Immortality'. It grows throughout Asian countries. It was first described in 1406 CE by Zhu Xiao in the book Materia Medica for Famine as useful survival food (9). Medical use of Jiaogulan has been recorded in 1578 in Li Shi-Zehen's Compendium of Materia Medica for the treatment of hematuria, edema in the pharynx and neck, tumors, and trauma (10). Today, Jiaogulan is appreciated as a medical plant with powerful biological effects. At the International Conference of TCM in Beijjing in 1991, Jiaogulan was declared to be one of 10 most effective medicinal plants used for a variety of as much as 54 diseases, conditions and syndromes, e.g., cardiovascular disease, hypertension, hepatitis, atherosclerosis, inflammation, and cancer. It is composed of a complex mixture of about 80 gypenosides, several amino acids, vitamins, and trace elements. Jiaogulan appertains to a class of plants called adaptogens. These herbs help the body to adapt to many forms of stress and imbalance. It exerts a unique two-way action on the central nervous system, calming the nerves when they are irritated and gently energizing them when they are depressed (11). Further beneficial effects attributed to GP are strong antioxidative activities (12), glucose lowering activities (13) and neuroprotective activity (14).

In the past we studied effects of GP on isolated mitochondria exposed to hypoxia/reoxygenation (15). There is increasing evidence that the mitochondrial phospholipid cardiolipin (CL) is an important component of oxidative phosphorylation mediating the transport of electrons along the respiratory chain and supporting ATP synthesis (16). Both the amount of CL and the composition of its molecular species affect the function of respiratory chain complexes and $\mathrm{F}_{0} \mathrm{~F}_{1}$-ATPase (17). It has been demonstrated that oxidation and subsequent degradation of molecular CL species can occur during ischemia/reperfusion $(18,19)$. We demonstrated a complete protection from permeabilization of the mitochondrial membrane system by GP (15). Moreover, GP prevented functional impairment of brain slices that were challenged with oxygen glucose deprivation even when GP was administered with reperfusion (20).

Indication for protective effects of GP in vivo has been provided previously (21). The authors demonstrated an attenuation of cognitive impairment at chronic cerebral hypo-perfusion in rats due to GP administration. Data regarding effects of GP on $\mathrm{CA} /$ resuscitation-mediated impairment of the CNS are, however, missing, although CA is one of the top three causes of death in the industrial world.

We tested the neuroprotective potential of GP in our well-established asphyxia induced CA (ACA) model in rat (22-24). In order to mimic the practical situation in health care in which intervention starts at best with but mostly after resuscitation, we applied GP simultaneously with resuscitation. In order to evaluate ACA-induced damage of the brain we examined vital parameters, novel object recognition as well as hippocampal cellular formation. Further, we focused on the impairment of mitochondria by evaluating amount and composition of CL, amount of mitochondria and activities of respiratory chain complexes.

\section{Materials and methods}

ACA model-animals and intervention protocol. Ethical approval for this study was granted according to the requirements of the German Animal Welfare Act on the Use of Experimental Animals and the Animal Care and Use Committees of Saxony-Anhalt (permit number 42502-2-2-947 Uni MD). Male rats (300-400 g; Institute's breeding population of inbred Wistar rats; Harlan-Winkelmann, Borchen, Germany; altogether 162; 128 included, 34 excluded, see below) were housed under controlled laboratory conditions

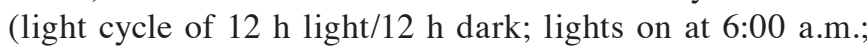
temperature, $20 \pm 2{ }^{\circ} \mathrm{C}$; air humidity, 55-60\%) with free access to water and chow. Every effort, including restriction to one single GP dose, was made to minimize the amount of suffering and the number of animals used in the experiments.

The study comprised of the following groups: i) Sham-operated; ii) sham-operated with DMSO applied once at the moment of spontaneous circulation re-establishment; iii) sham-operated with GP applied once at the moment of spontaneous circulation re-establishment; iv) ACA-treated; v) ACA-treated with DMSO applied once at the moment of spontaneous circulation re-establishment; vi) ACA-treated with GP applied once at the moment of spontaneous circulation re-establishment; vii) sham-operated with daily applied DMSO for 7 days; viii) sham-operated with daily applied GP for 7 days; ix) ACA-treated with daily applied DMSO for 7 days; and x) ACA-treated with daily applied GP for 7 days.

Groups i-vi consisted of 18 animals each; 5 for evaluation of mitochondrial parameters $6 \mathrm{~h}$ after ACA, 5 for evaluation of mitochondrial parameters $24 \mathrm{~h}$ after ACA, 3 for assessment of neurodegeneration $24 \mathrm{~h}$ after ACA, 5 for assessment of neurodegeneration 7 days after ACA; groups vii-x consisted of 5 animals each; altogether 128 animals. DMSO groups were installed to discriminate between GP effects and artificial effects of its solubilizer DMSO.

Anesthesia was induced with 5\% sevoflurane (Pfizer $\mathrm{GmbH}$, Berlin, Germany) in an oxygen/nitrous oxide mixture (40:60) via facemask followed by endotracheal intubation with a modified laryngoscope and a venous catheter and muscular relaxation with vecuronium (1 mg/kg; Pfizer). Mechanical ventilation was performed with intermittent positive pressure ventilation (IPPV). For drug administration, blood sampling, and continuous blood pressure monitoring both left femoral vessels were cannulated with polyethylene catheters. After 5 min of room air ventilation and baseline control, ACA was induced by an end-expiratory interruption of IPPV on paralyzed rats for $6 \mathrm{~min}$. ACA (defined as a non-pulsatile blood pressure of less than $10 \mathrm{mmHg}$ ) was reached within approximately 3 min (Fig. 1).

Resuscitation was performed by the administration of epinephrine (i.v.; $1 \mu \mathrm{g} / \mathrm{kg}$; Pfizer) and sodium bicarbonate ( $1 \mathrm{mEq} / \mathrm{kg}$ ), restarting IPPV with $100 \%$ oxygen for $1 \mathrm{~h}$, and manual external chest compression (200/min). Return of spontaneous circulation (ROSC) was defined as a pulsatile mean arterial pressure (MAP) above $60 \mathrm{mmHg}$. Rats with no ROSC within 2 min were excluded (altogether 34). The 2-min interval is our lab standard to avoid non-homogeneous ACA periods leading to pathophysiological differences and, subsequently, to the need for larger numbers of animals. Vital 


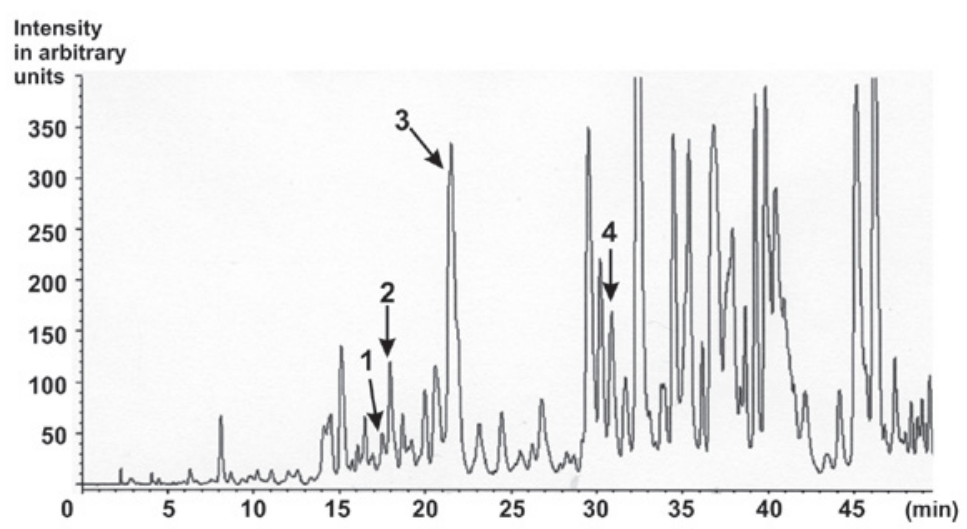

Figure 1. Characterisation of the ethanolic Gynostemma pentaphyllum extract. HPLC chromatogram of saponins from G. pentaphyllum extract with MS detection. Peak 1, Gypenoside LXIII (retention time, 16.373; content, 129.4 ppm); Peak 2, Gypenoside LXIII (retention time, 17.297; content, 316.4 ppm); Peak 3, Gypenoside IV (Ginsenoside $\mathrm{Rb}_{3}$; retention time, 20.497; content, 5899.5 ppm); Peak 4, Gypenoside VIII (Ginsenoside Rd; retention time, 29.813; content, $414.0 \mathrm{ppm}$ ) [as published in (25)].

parameters (ECG, blood pressure, temperature, airway pressure) were monitored continuously during the first $30 \mathrm{~min}$ of the post-resuscitation intensive-care phase. At 5, 15 and 30 min after ROSC, arterial blood samples were collected and evaluated for blood gases $\left(\mathrm{pCO}_{2}, \mathrm{pO}_{2}\right), \mathrm{pH}$ and glucose. After sufficient spontaneous respiration was established ( $\sim 30 \mathrm{~min}$ post ROSC), catheters were removed, cannulated vessels were ligated and incisions were surgically closed. Rats were extubated and returned to their cages. Body temperature was maintained at $37 \pm 0.3^{\circ} \mathrm{C}$ during preparation, insult and the first 30 min post-ROSC. Thereafter, normal body temperature was stably maintained within normal range by placing the rats in an incubator cage for $24 \mathrm{~h}$.

Gynostemma pentaphyllum (GP)/DMSO intervention. The standardized GP powder, obtained by extraction of dried aerial parts with $75 \%$ ethanol $/ 25 \%$ water, was received from Herbasin Co., Ltd. (Shenyang, China; batch no. 061001E074). The extract composition was certified by the manufacturer. According to the manufacturer, the extract contained a gypenoside content of $99.7 \%$, a heavy metal contamination $<10 \mathrm{ppm}$ and no microbiological contamination. In the HPLC-fingerprint performed by the consumer, eight peaks corresponding to gypenosides were detected (15). In particular, Gypenoside LXIII, Gypenoside $\mathrm{Rb}_{3}$ and Gypenoside VIII could be identified (Fig. 1) (25). The retention times of specific gypenosides were determined by using standard gypenosides in separate experiments. The respective method has been described in detail previously (25). Briefly: Sample preparation: $5 \mathrm{mg}$ of the dried ethanolic extract were dissolved in $1 \mathrm{ml}$ of ethanol, filtered over Millipore ${ }^{\circledR}$ filtration unit, type $0.45 \mu \mathrm{m}$, and injected into the HPLC apparatus. Injection volume: Gynostemma pentaphyllum extract: $20.0 \mu \mathrm{l}$. HPLC parameter: Apparatus: MERCK HITACHI D-6000 A Interface, MERCK HITACHI AS-2000 Autosampler, MERCK HITACHI L-6200 A Intelligent Pump. Separation column: LiChroCART ${ }^{\circledR}$ 250-4 LiChrospher ${ }^{\circledR} 100$ RP-18 (5 $\left.\mu \mathrm{m}\right)$ (Merck). Precolumn: LiChroCART $^{\circledR}$ 4-4 LiChrospher ${ }^{\circledR} 100$ RP-18 (5 $\left.\mu \mathrm{m}\right)$ (Merck). Solvent: A: dist. Water (Millipore Ultra Clear UV plus ${ }^{\circledR}$ filtered), B: acetonitrile (Fa. VWR). Gradient: 5-100\% B in $60 \mathrm{~min}$, total runtime: $60 \mathrm{~min}$. Flow: $0.8 \mathrm{ml} / \mathrm{min}$. A single quadruple mass spectrometer (LC/MS) with electrospray ionization (ESI) in negative mode was used for detection (26).

GP powder $(60 \mathrm{mg})$ was dissolved in $0.5 \mathrm{ml} \mathrm{DMSO}$ and diluted with PBS (phosphate-buffered saline) to a final volume of $10 \mathrm{ml}$. In pilot studies a volume of $200 \mu \mathrm{l}$ of this solution injected i.p. was found to be optimal for fast resorption and distribution within the circulation.

The tolerability and efficacy of GP was tested in vitro using both cultures of dispersed astrocytes and hippocampal slices (20). A dosis of $60 \mu \mathrm{g} / \mathrm{ml}$ was found to be optimal in vitro. The applied $200 \mu \mathrm{l}$ of the stock solution contained $1,200 \mu \mathrm{g}$ GP. At an average animal weight of about $350 \mathrm{~g} / \mathrm{a}$ blood volume of about $20 \mathrm{ml}$ that resulted in the intended final concentration of $60 \mu \mathrm{g} / \mathrm{ml}$.

As vehicle control a corresponding DMSO solution was injected. First dose of GP/DMSO was administered at the time when spontaneous circulation was re-established. In groups vii-x, GP or DMSO were additionally administered every morning for the next 6 days.

Preparation of tissue homogenates. After the respective survival times, animals were sacrificed by over-dosed anesthesia (isoflurane $10 \%$ in a in a 4.351 sealed desiccator; Baxter, Unterschleissheim, Germany). Brains were quickly removed and hippocampi were separated on ice, weighed, minced using small scissors, transferred into ice-cold phosphate buffer solution (PBS; pH 7.4; $10 \%$ tissue portion) and homogenized at $4^{\circ} \mathrm{C}$ using a Potter-Elvehjem glass-Teflon homogenizer (10 strokes at $600 \mathrm{rpm}$ ). The mitochondrial parameters citrate synthase activity, NADH:cytochrome c oxidoreductase activity, succinate:cytochrome c oxidoreductase activity and cardiolipin were determined by using the homogenates. The data were related either to mg protein of the homogenate or to the activity of the mitochondrial marker enzyme citrate synthase.

Determination of citrate synthase activity (EC 2.3.3.1). Citrate synthase (CS) activity was assayed in homogenates of hippocampi 6 and $24 \mathrm{~h}$ after ACA using a standard procedure at $30^{\circ} \mathrm{C}(27)$. Before running the assay samples were fivefold frozen and defrosted to permeabilize membranes. Homogenates of $40 \mu \mathrm{g}$ were evaluated. The increase of CoA 
absorption was monitored at $412 \mathrm{~nm}$ with a Cary 100 Bio spectrophotometer (Varian, Darmstadt, Germany).

Determination of NADH:cytochrome c oxidoreductase activity (EC 1.6.99.3). The parameter was assayed in homogenates of hippocampi 6 and $24 \mathrm{~h}$ after ACA. Samples $(500 \mu \mathrm{l})$ of the respective tissue homogenates were fivefold frozen and defrosted to permeabilize membranes. A volume of $15 \mu 1$ (40 $\mu \mathrm{g}$ protein) was used to run the standard assay at $30^{\circ} \mathrm{C}$. The reduction of cytochrome $\mathrm{c}$ was followed by measuring the absorption at $550 \mathrm{~nm}$ with a Cary 100 Bio spectrophotometer (Varian). The rotenone-sensitive absorption was used for quantification.

Determination of succinate:cytochrome c oxidoreductase activity (EC 1.3.5.1). The parameter was assayed in homogenates of hippocampi 6 and $24 \mathrm{~h}$ after ACA. Samples $(500 \mu 1)$ of the respective tissue homogenates were fivefold frozen and defrosted to permeabilize membranes. A volume of $15 \mu \mathrm{l}\left(40 \mu \mathrm{g}\right.$ protein) was used to run the standard assay at $30^{\circ} \mathrm{C}$. The reduction of cytochrome $\mathrm{c}$ was followed by measuring the absorption at $550 \mathrm{~nm}$ with a Cary 100 Bio spectrophotometer (Varian). The antimycin A-sensitive absorption was used for quantification.

Assessment of cardiolipin (CL). CL was also analyzed in hippocampi $24 \mathrm{~h}$ after ACA. Therefore, samples (see above) were frozen on dry ice and stored at $-80^{\circ} \mathrm{C}$ until CL analysis, steps of which were i) extraction of CL, $50 \mathrm{ng}$ of tetra-myristoyl-CL [(C14:0 $)_{4}$-CL; Avanti Polar Lipids Inc., Alabaster, AL, USA; internal standard] and $4.2 \mathrm{ml}$ chloroform/methanol (2/1, $\mathrm{v} / \mathrm{v}$ ) containing $0.05 \%$ butylated hydroxytoluene (BHT; Avanti Polar Lipids Inc.) were added to $100 \mu 1$ of defrosted tissue homogenates. The lipid and aqueous phases were separated by adding $800 \mu \mathrm{l}$ of $0.01 \mathrm{M} \mathrm{HCl}$, intensive shaking and subsequent centrifugation. After centrifugation, the lipid phase (lower phase) was collected and dried under nitrogen atmosphere and acidified. Ice-cold methanol $(2 \mathrm{ml})$, chloroform $(1 \mathrm{ml})$ and $1 \mathrm{ml}$ of $0.1 \mathrm{M} \mathrm{HCl}$ were added. The solution was intensively mixed. After 5 min of incubation on ice the samples were separated by the addition of chloroform $(1 \mathrm{ml})$ and $0.1 \mathrm{M} \mathrm{HCl}(1 \mathrm{ml})$. The chloroform/methanol phase was recovered as CL-containing sample. Afterwards, the samples were dried under nitrogen and dissolved in $0.8 \mathrm{ml}$ chloroform $/ \mathrm{methanol} /$ water $(50 / 45 / 5, \mathrm{v} / \mathrm{v} / \mathrm{v})$. After mixing and filtering of the mixture over $0.2 \mu \mathrm{m}$ PTFE membranes the samples were ready for use. ii) HPLC-MS/MS analysis as described in detail earlier (28).

Determination of protein. The protein content was determined according to the Bradford method (29) using bovine serum albumin as the standard.

Assessment of brain degeneration. After survival times of $24 \mathrm{~h}$ and 7 days, anaesthetized rats were sacrificed by transcardial perfusion with $4 \% 0.1 \mathrm{M}$ phosphate-buffered paraformaldehyde (PFA; pH 7.4; Millipore, Darmstadt, Germany). The brains were quickly removed, post-fixed in the same fixative at $4^{\circ} \mathrm{C}$ overnight, cryoprotected in $30 \%$ sucrose in $0.4 \%$ PFA (pH 7.4) for 2 days, and rapidly frozen at $-20^{\circ} \mathrm{C}$. Slice preparation and staining procedure were performed as described (24).
The following mixtures of primary antibodies (diluted in $10 \%$ fetal calf serum and $0.3 \%$ Triton-X 100 in PBS) were used: (i) Mouse monoclonal anti-NeuN (neuronal nuclei antibody; Chemicon, Billerica, USA; 1:100) and polyclonal rabbit anti-glial fibrillary acidic protein (GFAP; Progen, Heidelberg, Germany; 1:500), and ii) monoclonal mouse anti-MAP2 (microtubule-associated protein 2; Covance, Münster, Germany; 1:1,000); and rabbit polyclonal anti-ionized calcium binding adaptor molecule 1, (IBA1; Abcam, Cambridge, UK; 1:1,000). The mixture of the secondary antibodies consisted of goat anti-mouse Alexa 488 (green; Invitrogen, Carlsbad, USA; 1:500) and donkey anti-rabbit Cy3 (red; Dianova, Hamburg, Germany; $1: 500$ ) and was diluted in $1 \%$ normal goat serum and $0.3 \%$ Triton- $X$ in PBS.

Using an AxioImager.M1 fluorescence microscope (Zeiss, Jena, Germany; with a Plan-Neofluar fluorescein/rhodamine objectives) slices were evaluated. For MAP2 analysis, five alternating slices/animal were scanned image field by image field (objective $x 40 / 0.75$ ) to compose an image of the complete hippocampus (1,388x900 pixel; AxioVision software 'Panorama', Zeiss). After that a standard evaluation window (500x300 pixel), including the complete hippocampal CA1 region, was selected manually and the respective staining intensity was quantified using ImageJ software (http:/rsbweb. nih.gov/ij/).

For quantification of pyknotic ( $24 \mathrm{~h}$ post-intervention) and normal NeuN-positive (7 days post-intervention) cells of the CA1 region, the AxioVision z-stack software (Zeiss) was used to get a composition image from 5-8 single images (objective $x 40 / 0.75$ ) taken at different focal distances of the slice. All clearly recognizable pyknotic/hyperchromatic cells and NeuN-positive cells were counted unbiased.

GFAP and IBA1 immunostainings of the hippocampal CA1 region were only descriptively analyzed.

For each antibody, microscopic settings and the exposure time of the fluorescence channels were set on the basis of control slices and kept equal for the corresponding preparation.

Novel object recognition test. After 7 days of survival, rats underwent the object recognition test in an open field (clear Plexiglas, $50 \mathrm{~cm}$ wide $\mathrm{x} 25 \mathrm{~cm}$ high) combined with a video tracking system (VPC-FH1; Sanyo Electric, Moriguchi, Japan). The experiment started with an adaptation trial without object in the open field, followed by two sessions, a sampling and a test trial, each 6 min long. In the sampling session, rats explored one object (A1, glass bottle $12 \mathrm{~cm}$ tall, $5 \mathrm{~cm}$ wide). The test session was conducted $120 \mathrm{~min}$ later by allowing rats to explore object A (now A2) together with a novel object (B, metal box, $5 \mathrm{~cm}$ tall $\mathrm{x} 5 \mathrm{~cm}$ wide). Thereby object $A$ was placed at the same place as in the first session. Each session was video recorded for later analysis. Three independent experimenters, blinded to group treatment, scored each behavioral test. Exploration was defined as sniffing or touching the object with the nose. Longer exploration duration of object B vs. object A2 can be interpreted as evidence for an intact recognition memory (30). Moreover, global habituation (comparison of the total time spent in exploring object A1 during the sampling session to the summarized total time spent in exploring objects A2 and B in the test phase) was determined. Data are presented as mean \pm SD. 

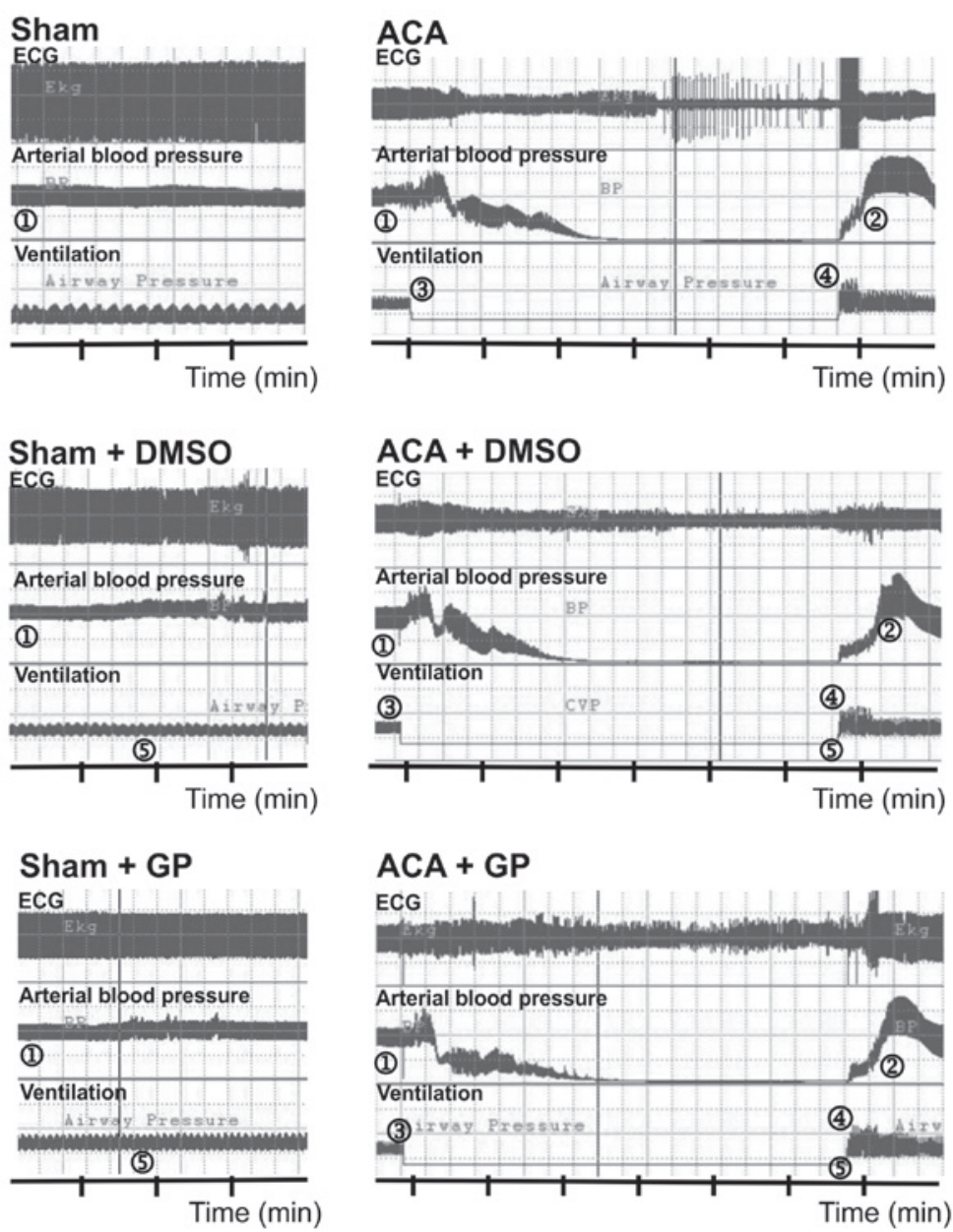

Figure 2. Examples of cardiovascular response to ACA/resuscitation. 1, MAP $\sim 120 \mathrm{mmHg} ; 2, \mathrm{MAP} \sim 200 \mathrm{mmHg}$; 3 , stop of mechanical ventilation and start of asphyxia; 4, start of cardio pulmonary resuscitation; 5 , time point of possible intervention with DMSO or GP. GP, Gynostemma pentaphyllum; DMSO, dimethyl sulfoxide; ECG, electrocardiogram.

Statistical analysis. All quantitative data are presented as the mean \pm SD per animal. The means (the exact $n$ is given in the respective figure legends) were analyzed with the non-parametric Kruskal-Wallis test and the Dunn's multiple comparison post-hoc test using Graph Pad Prism 6 (GraphPad Software Inc., La Jolla, CA, USA). For intra-group differences of sham- and ACA-animals one-way ANOVA was performed. Differences of groups with equal treatment regimens but different survival times were analyzed with the Wilcoxon-Mann-Whitney-test. In either case $\mathrm{P} \leq 0.05$ was considered to indicate a statistically significant difference.

\section{Results}

Effect of ACA and DMSO/GP on vital parameters. The preparation of animals including anesthesia via facemask, trachea intubation, cannulation of the left femoral vessels and determination of baseline parameters required $22 \pm 5$ min. Fig. 2 illustrates the recording of ECG, arterial blood pressure and ventilation. In sham-operated animals, DMSO/GP intervention did not influence ECG and blood pressure pattern. In ACA animals, arterial blood pressure dropped to null line after $170 \pm 28 \mathrm{sec}$ of asphyxiation without statistical difference between the three ACA groups. Shortly after stop of ventilation, the ECG signal amplitude became suppressed with remarkable fluctuation. It proceeded to asystole within $180 \pm 50 \mathrm{sec}$. The recording was aggravated by typical technical transients. When 6 min of asphyxiation were completed, resuscitation was initiated. Thereby, ROSC was achieved within $30 \pm 24 \mathrm{sec}$. Independent of DMSO/GP intervention, resume of ECG activity started with an initial pattern resemblant to burst-suppression.

The time course of the basic vital parameters is displayed in Fig. 3. ACA induced a transient increase of MAP (hypertensive in tendency $1 \mathrm{~min}$ after ACA but, not significant, $\mathrm{P}=0.07$ returning to normal values afterwards; Fig. 3A), a significant increase of heart rate (Fig. 3B), of arterial carbon dioxide tension ( $\mathrm{pCO}_{2}$; Fig. 3C) and of blood glucose values (Fig. 3E), whereas $\mathrm{pH}$ levels were significantly reduced (Fig. 3F). In the presence of GP, the ACA-mediated increase of $\mathrm{pCO}_{2}$ (Fig. 3C) and blood glucose concentration (Fig. 3E) was prevented. DMSO was able to normalize both ACA-mediated increases too (Fig. 3C and E).

Preparation of the animals did not affect these vital parameters; all parameters were within the physiological ranges (baseline). The arterial $\mathrm{pO}_{2}$ of ACA-animals was increased after $30 \mathrm{~min}$ as consequence of ventilation with $100 \%$ oxygen (Fig. 3D). The body temperature (tympanal and rectal) was not affected within the 30 min-monitoring period after resuscitation (data not shown). 

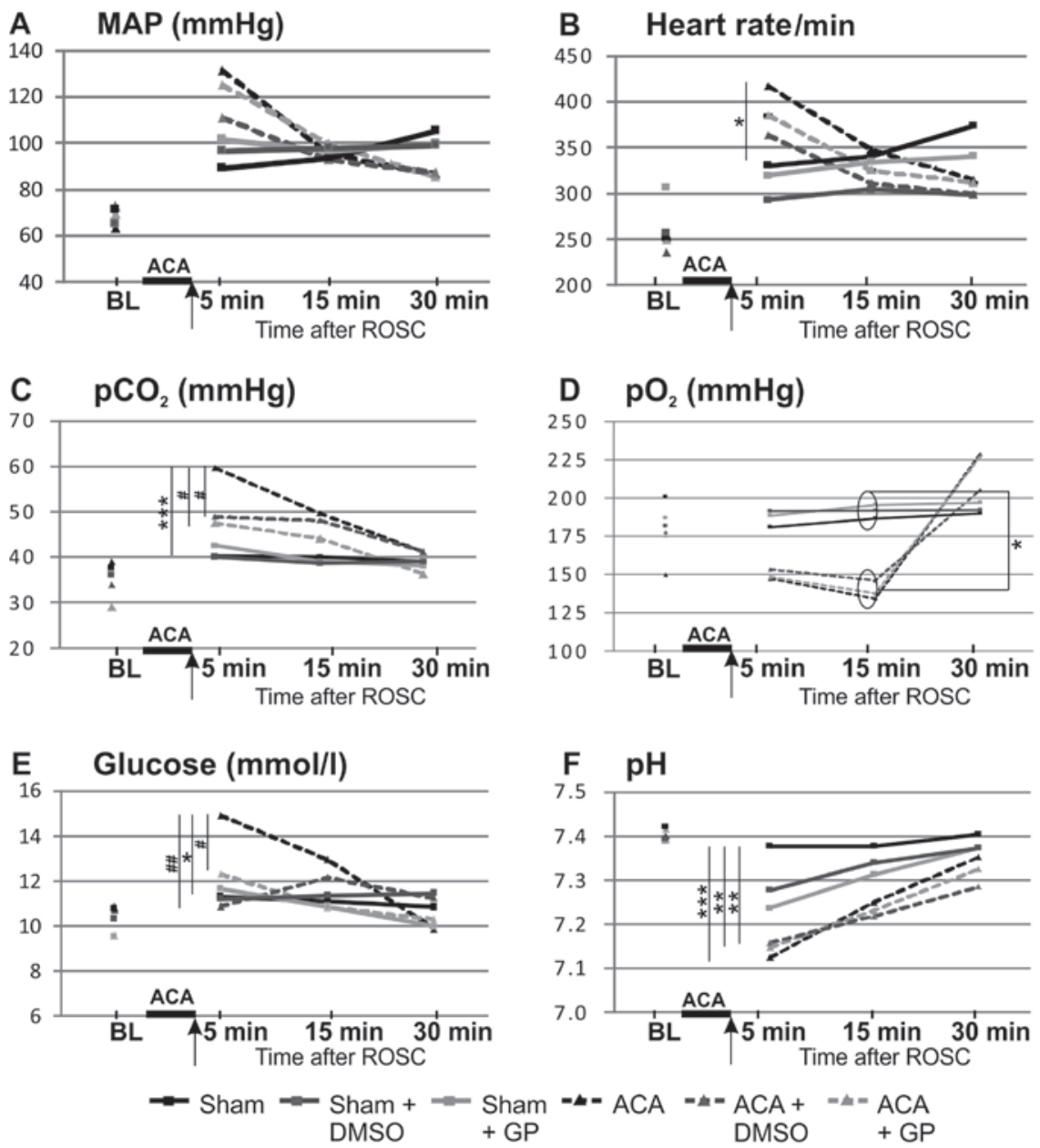

Figure 3. Pre- and post-resuscitation physiological parameters: (A) MAP, (B) heart rate, (C) $\mathrm{pCO}_{2}$, (D) $\mathrm{pO}_{2}$, (E) glucose and (F) pH. Arrow, time point of DMSO/GP intervention. Data: Mean \pm SD with $\mathrm{n} \geq 15$; Kruskal-Wallis/Dunn's post-hoc test; significant differences sham vs. ACA: ${ }^{*} \mathrm{P}<0.05$ and ${ }^{* * * *} \mathrm{P}<0.001$ within the ACA groups; ${ }^{~} \mathrm{P}<0.05$ and ${ }^{\# \#} \mathrm{P}<0.005$ within the sham groups, yet no significant differences were found. $\mathrm{BL}$, base line; $\mathrm{MAP}$, mean arterial pressure; $\mathrm{pCO}_{2}$, arterial carbon dioxide tension; $\mathrm{pO}_{2}$, arterial oxygen tension, ACA, asphyxia cardiac arrest; GP, Gynostemma pentaphyllum; ROSC, return of spontaneous circulation.; DMSO, dimethyl sulfoxide.

Effect of ACA and DMSO/GP on amount of mitochondria and activity of respiratory chain complexes. We used the activity of CS as marker for the amount of mitochondria in order to study changes in mitochondrial mass. In sham animals, $6 \mathrm{~h}$ after treatment (early response) increased values of CS were found in animals exposed to DMSO as the vehicle control (Fig. 4A). However, in combination with GP no effect was observed (Fig. 4A). Twenty four hours after treatment (late response), no effect of DMSO could be detected in sham animals (Fig. 4B). In ACA treated animals, DMSO did not cause changes in CS activity at all (Fig. 4A, B). GP caused increase in CS activity only in ACA animals at both time points (Fig. 4A, B).

In order to explore the effect of ACA on the expression of respiratory chain complexes in mitochondria the activities of $\mathrm{NADH}$-cytochrome c oxidoreductase (complex I + III) and succinate:cytochrome c oxidoreductase (complex II + III) were analyzed. For clear interpretation of the data, the complex activities were related to protein content of homogenate samples (Fig. 4C, D, G, H) or to CS activities representing the mitochondrial amount in the homogenate (Fig. 4E, F, I, J). When complex I + III activities were related to $\mathrm{mg}$ protein of homogenate samples similar results of GP as for citrate synthase activities were obtained (Fig. 4C, D). The specific complex I + III activities (related to CS activity) displayed no singular DMSO or GP effect. $6 \mathrm{~h}$ after treatment, we detected increased specific activities of complex I + III of all ACA-treated animals in comparison to the respective sham operated groups (Fig. 4E). Twenty four $\mathrm{h}$ after treatment, the specific complex I + III activities of all sham animals were in tendency elevated possibly indicating a stress response to anesthesia (Fig. 4F).

The analysis of complex II + III activities revealed that DMSO caused decrease in complex activity in sham operated and in ACA-treated animals $6 \mathrm{~h}$ post ACA as a side effect (Fig. 4G, I). This effect declined with survival time (Fig. 4H, J). ACA alone caused increase in complex II + III activity $6 \mathrm{~h}$ after treatment (Fig. 4G,I) that was not detected in the presence of GP. Moreover, GP neutralized the side effect of DMSO (Fig. 4G, I).

Content and composition of the mitochondrial phospholipid $C L$. To evaluate the CL tissue content the amount of CL was related to $\mathrm{mg}$ protein of homogenate. The corresponding data are presented in Fig. 5A. For the determination of the specific mitochondrial content of CL the data were related to the activity of the mitochondrial marker enzyme CS. 
$6 \mathrm{~h} \quad$ Hippocampus

$24 \mathrm{~h}$
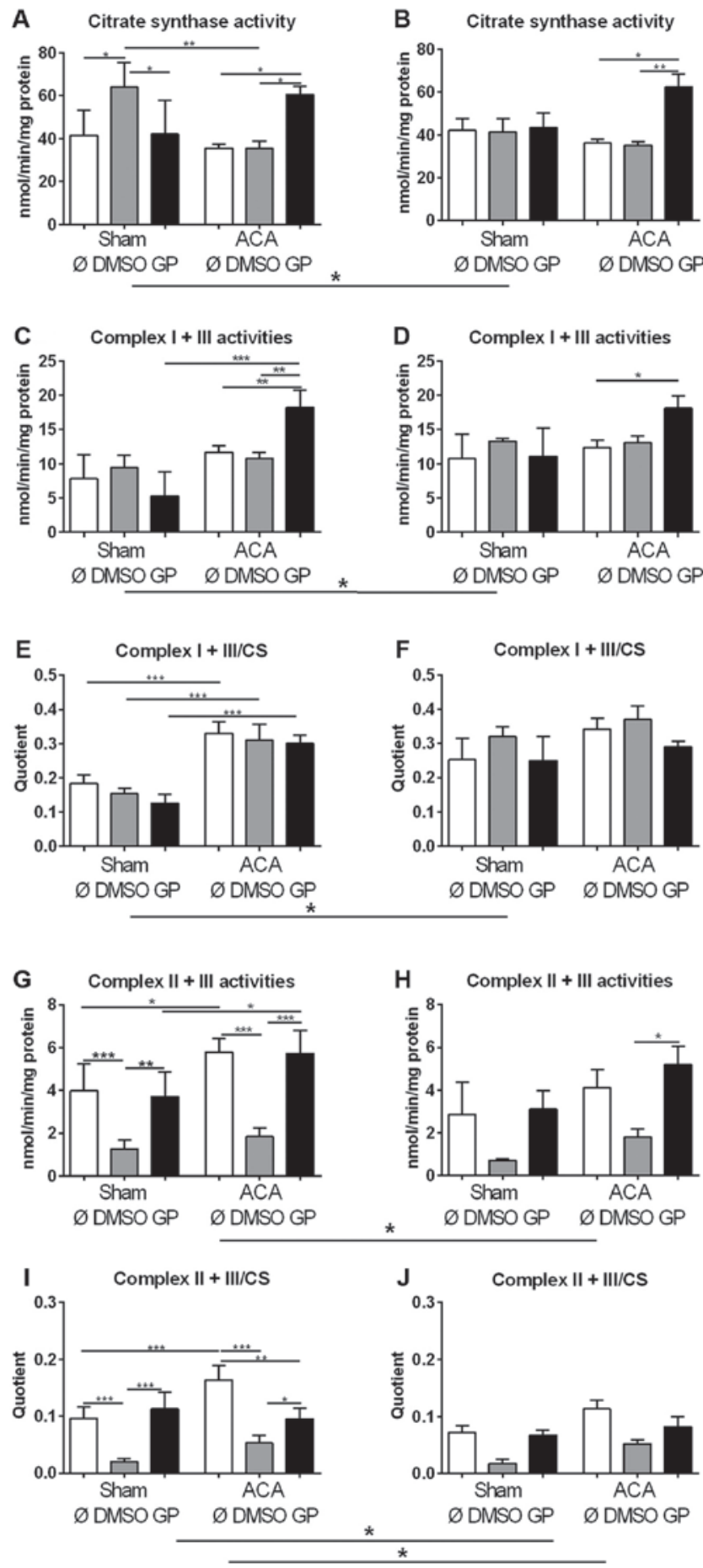

Figure 4. Effect of ACA on mitochondrial content and respiratory chain complex activities. (A and B) Citrate synthase (CS) activities and specific activities of the respiratory chain complexes I + III (C and D) and II + III (G and $\mathrm{H})$ were determined in hippocampus after $6 \mathrm{~h}(\mathrm{~A}, \mathrm{C}, \mathrm{E}, \mathrm{G}$ and I) and $24 \mathrm{~h}(\mathrm{~B}$, $\mathrm{D}, \mathrm{F}, \mathrm{H}$ and J). GP and the vehicle control DMSO were administered with resuscitation. Specific respiratory chain complex activities were calculated by relating the data to CS activities (E, F, I and J). Data are expressed as mean \pm SD from 5 animals. Significance of differences was tested with Kruskal-Wallis/Dunn's post-hoc test; $\left({ }^{*} \mathrm{P}<0.05 ;{ }^{* *} \mathrm{P}<0.005,{ }^{* * *} \mathrm{P}<0.001\right)$. ACA, asphyxial cardiac arrest; GP, Gynostemma pentaphyllum; DMSO, dimethyl sulfoxide.

These data are presented in Fig. 5B. ACA caused significant increase in both the tissue content of CL and in the mitochondrial CL content. The stimulation of CL synthesis by
A
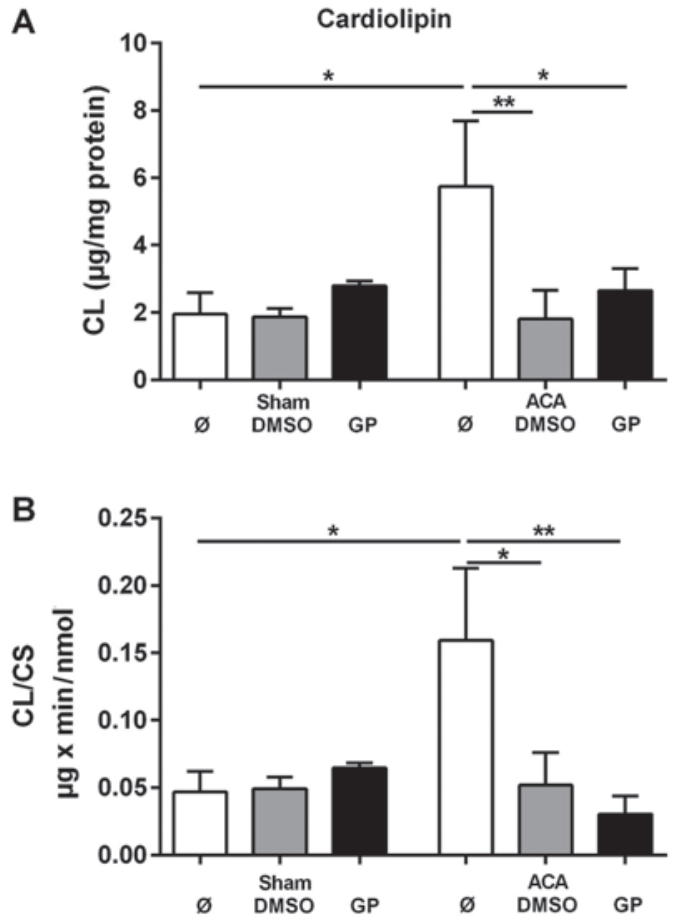

Figure 5. Effect of ACA on mitochondrial CL content. CL content was determined in hippocampus after $24 \mathrm{~h}$. GP and the vehicle control DMSO were administered with resuscitation. (A) $\mathrm{CL}$ content in absolute values. (B) Mitochondrial CL contents were calculated by relating the data to citrate synthase activities (See Fig. 3A and B). Data are expressed as mean \pm SD from 5 animals. Significance of differences was tested with Kruskal-Wallis/Dunn's post-hoc test, ( $\left(\mathrm{P}<0.05 ;{ }^{* *} \mathrm{P}<0.005\right)$. CD, cardiolipin; GP, Gynostemma pentaphyllum; DMSO, dimethyl sulfoxide; ACA, asphyxial cardiac arrest.

ACA was prevented by the administration of DMSO and GP, respectively. We did not find any change in the composition of molecular CL species under the conditions of investigation (data not shown).

Histological outcome of hippocampal CA1 region $24 \mathrm{~h}$ post $A C A$. In sham animals, the NeuN-immunostaining showed intact hippocampal CA1 neurons (Fig. 6A). The MAP2-immunostained fiber net (Fig. 6B) was well developed. The patterns of GFAP-positive astroglia (Fig. 6A) or IB1-positive microglia (Fig. 6B) conformed to norms. In DMSO-treated sham animals, a couple of pyknotic cells was seen in the CA1 pyramidal cell layer (Fig. 6C, arrows). Pyknotic cells are characterized by size-reduction and/or condensation usually associated with hyperchromatosis, that has been demonstrated by NeuN immunostained nuclei. It indicated cell death induction by DMSO, which was confirmed by MAP2 staining, offering an ongoing loosening up of the nerve fiber net (Fig. 6D). The pattern of astroglia (Fig. 6C) and microglia (Fig. 6D) showed signs of activation. The respective staining patterns of GP-treated sham animals were identical to those of the untreated group (data not shown).

In the hippocampal CA1 pyramidal cell layer of ACA treated animals (Fig. 6E), a massive increase of pyknotic cells (arrows) as well as first cell losses (arrowheads) were found. The respective MAP2-positive fiber nets revealed distinct signs of disruption (Fig. 6F). Yet, astroglia activation was not evidenced by GFAP immunostaining (Fig. 6E). Activation of microglia 

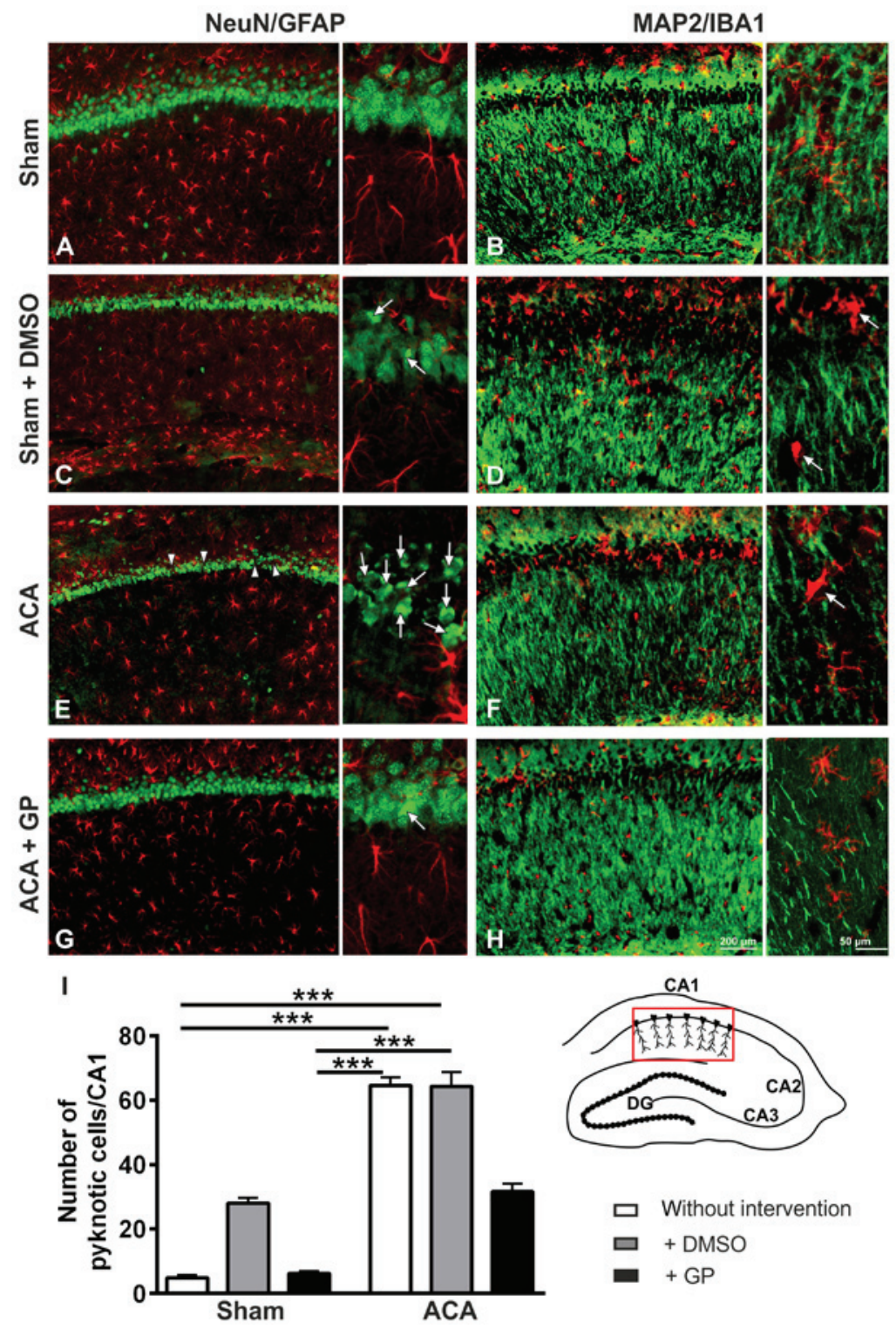

Figure 6. Representative fluorescence images of rat hippocampal CA1 pyramidal cell layer $24 \mathrm{~h}$ post intervention. For better orientation please refer to the hippocampal scheme below the microphotographs. The CA1 region is highlighted in red. (A) Sham-operated animals with normal pattern of NeuN-positive (green) neurons and GFAP-positive astroglial cells (red). (B) Sham-operated animals with regularly formed MAP2-positive (green) fiber networks in the strata radiatum and oriens and normally distributed ramified IBA1-positive microglial cells (red). (C) Sham-operated animals with DMSO intervention offering first signs of neuronal pyknosis (arrows). The GFAP-related astroglia state seem to be, however, unchanged. (D) Sham-operated animals with DMSO intervention offering first signs of fiber net break up. The IBA1-positive microglia offer signs of activation by starting to change from ramified to amoeboid form (arrows). (E) ACA-animals with pyknotic cells (arrows) and some neuronal loss (arrowheads), whereas the astroglial pattern still remains sham-like. (F) ACA-animals with disrupted fiber net (MAP2) and activated IBA1-stained microglia (arrow). (G) ACA-animals with GP intervention offering a reduced number of pyknotic cells (arrow) at unchanged astroglial state. (H) ACA-animals with GP intervention offering a less scattered fiber net (MAP2) and a mostly ramified microglia. (I) Semi-quantitative analysis of pyknotic NeuN-positive neurons; data are mean \pm SD; Kruskal-Wallis/Dunn's post-hoc test; $\mathrm{n}=3$ animals/group; ${ }^{* * *} \mathrm{P}<0.001$. ACA, asphyxia cardiac arrest; GP, Gynostemma pentaphyllum; DMSO, dimethyl sulfoxide; the given bars apply for all respective pictures.

was, however, clearly demonstrable; the cell bodies became more rounded and the originally ramified branches became shorter and stockier (arrow in Fig. 6F). The respective staining patterns of DMSO-treated ACA-animals were identical to those of the untreated ACA group (data not shown). In case of GP administration, neuroprotective tendencies were found: lower number of pyknotic neurons (arrows in Fig. 6G) and less disruption of the MAP2-positive fiber net (Fig. 6H). Semi-quantification of pyknotic NeuN-positive cells is given in Fig. 6I.

Histological outcome of hippocampal CA1 region 7 days post-ACA. In untreated sham-operated animals, NeuN
(Fig. 7A) as well as MAP2 (Fig. 7B) immunostaining revealed intact hippocampal CA1 neurons with a dense fiber network. A consistent standard pattern of GFAP-positive astroglia (Fig. 7C) and IB1-positive microglia (Fig. 7D) was also evident. In both, onetime (shown in Fig. 7E-H) or multiple (not shown) DMSO-treated sham-animals, the NeuN-stained CA1 pyramidal cell layer showed a spotty cell loss (arrows in Fig. 7E). These areas offered a narrow accumulation of IBA1-positive microglia cells (arrows in Fig. 7H). MAP2 (Fig. 7F) and GFAP (Fig. 7G) immunostainings revealed, however, regular patterns. In GP treated sham-animals, there were no signs of abnormal neuronal viability or cell pattern, 

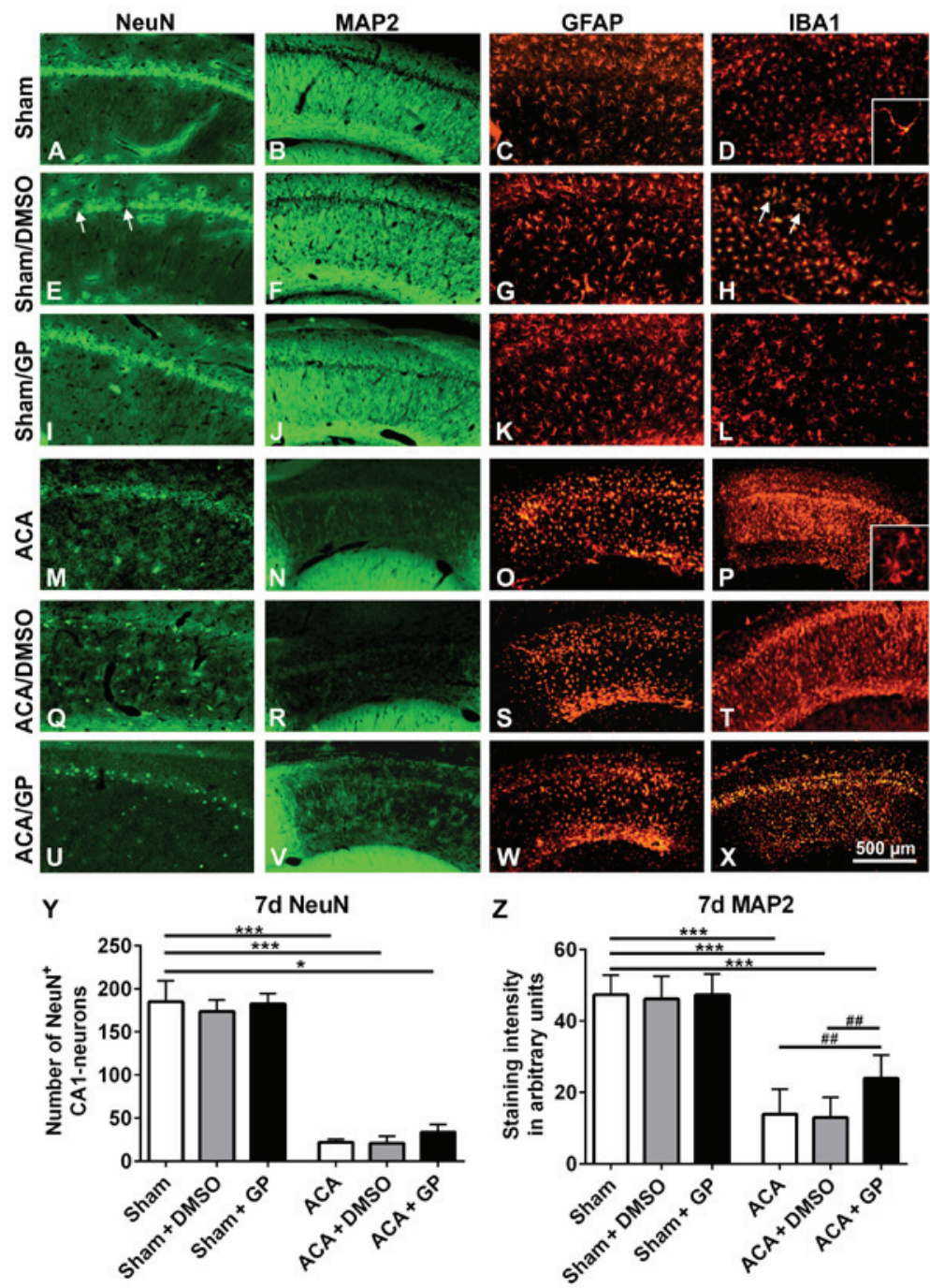

Figure 7. Representative fluorescence images of rat hippocampal CA1 pyramidal cell layer 7 days post-intervention. In untreated sham-operated animals, the CA1 pyramidal cell line (A) NeuN, the respective fiber network (B) MAP2, the astroglia pattern (C) GFAP, and the microglia shape (D) IBA1 were normal. Intervention with onetime applied DMSO induced a spotty loss of pyramidal cells (arrows in E). The respective fiber network (F) and the astroglia shape (K) showed, however, no abnormalities. IBA1 staining offered a limited microglia accumulation in the region of damaged neurons (arrows in H). In GP treated sham-animals, the CA1 pyramidal cell line $(\mathrm{I})$, the respective fiber network $(\mathrm{J})$, the astroglia pattern $(\mathrm{K})$, and the microglia shape $(\mathrm{L})$ were again properly formed ACA induced a massive loss of CA1 pyramidal cells. The neuronal cell loss was indicated by the distinct reduction of NeuN immunofluorescence signal (M) as well as by the massive reduction of MAP2 staining of the respective nerve fiber network (N). Consistently, significant activation of astroglia, indicated by massive upregulation of GFAP staining $(\mathrm{O})$, and activation of microglia, indicated by upregulation of IBA1 immunofluorescence signal (P) were seen. Thereby, microglia conversed from ramified to amoeboid cell morphology (insert D vs. insert P). (Q-T) Onetime applied DMSO treatment did not further amplify these ACA-induced signs of neurodegeneration. (U-X) Onetime applied GP counteracted these ACA-induced processes to some extent. NeuN-positive CA1 neurons were partly preserved (U) and also some MAP2-positive nerve fibers survived (V). Patterns of astroglia (W) and microglia (X) were, however, equally sever pronounced as in ACA and ACA-DMSO animals. (Y) Semi-quantification of NeuN-positive CA1 neurons. (Z) Semi-quantification of MAP2-stained CA1 fibers. Data are mean $\pm \mathrm{SD}$; Kruskal-Wallis/Dunn's post-hoc test $\left({ }^{*} \mathrm{P}<0.05 ;{ }^{* * *} \mathrm{P}<0.001\right)$ and one-way ANOVA for intra-group differences of ACA-animals $\left({ }^{* \#} \mathrm{P}<0.005\right)$; all with n=5/group, ACA, asphyxia cardiac arrest; GP, Gynostemma pentaphyllum; MAP2, microtubule-associated protein 2; NeuN, neuronal nuclei; GFAP, glial fibrillary acidic protein; IBA1, ionized calcium binding adaptor molecule 1; DMSO, dimethyl sulfoxide; the given bar applies for all pictures.

irrespective of whether GP was applied once or multiple (Fig. 7I-L, demonstrated for onetime applied GP). That indicates that GP was able to counteract cell stress mediated by the vehicle DMSO.

In ACA-animals, the hippocampal NeuN-stained CA1 pyramidal cells were massively degenerated (Fig. 7M). In parallel, MAP2 immunostained nerve fibers showed a massive failure (Fig. 7N). Activation of GFAP-positive astroglia (Fig. 7O) and IBA1-positive microglia (Fig. 7P) was clearly evident. That was demonstrated by enhanced immunoreactivity of the resident glia cells. Moreover, microglia migration as well as conversion from ramified to amoeboid cell morphology took place (insert Fig. 7D vs. insert 7P). DMSO intervention, irrespective of its application regime, did not further impair these patterns (Fig. 7Q-T; demonstrated for onetime applied DMSO). GP, independently of its application regime, was able to counteract these degenerative changes to some degree; the band of NeuN-positive CA1 neurons seemed to be partly retained (Fig. 7U, demonstrated for onetime applied GP) and some of MAP2-positive fibers survived (Fig. 7V). Activation of astroglia (Fig. 7W) as well as microglia (Fig. 7X) was as high as in ACA and ACA-DMSO animals. Obviously, the strong neurodegenerative potential of ACA limited the GP-mediated neuroprotection. Semi-quantification is given for 
NeuN-positive CA1 neurons in Fig. 7Y and for MAP2-stained CA1 fibers in Fig. $7 Z$.

Novel object recognition test. Sham-operated rats, independent of non-/DMSO-/GP-treatment, were able to discriminate between a 'known' and a novel object; the exploration time of object B at simultaneous presence of object A (A2) was roughly twice as long (Fig. 8A). Moreover, the totalized exploration time for A2 plus B in the test trial was significantly higher than for object A alone (A1) in the sampling trial (Fig. 8B).

The recognition ability of ACA-stressed animals without DMSO- or GP-intervention was, actually unexpected, unchanged when compared with sham-animals. The exploration time difference (B vs. A2) was even more pronounced (Fig. 8A). Now, this pattern was moderately affected by DMSO and dramatically by GP. DMSO-treated animals seemed to be better in the recognition of the 'known' object A (A1 vs. A2 significantly different; $\mathrm{P}<0.05)$. This effect of better recognition, even more distinctive $(\mathrm{P}<0.001)$, was also found in GP-treated rats. Additionally, the sampling behavior of the GP-treated ACA-stressed animals was extremely pronounced. They explored object A1 significantly longer than animals from all other groups (Fig. 8A). And the GP-treated animals were the only group with significant shorter $(\mathrm{P}<0.05)$ exploration time for the novel object $\mathrm{B}$ when compared with the exploration time of object $\mathrm{A}$ at the first session.

As demonstrated in Fig. 8B, the GP-effect in ACA-animals was strong enough to completely reverse the significant difference of the global habituation pattern. In the test trial (object A2 $+\mathrm{B}$ ), the exploration time of GP-treated ACA-animals was significantly lower $(\mathrm{P}<0.05)$ then the time they spend to explore object A1 in the first session. DMSO treatment of ACA-animals reduced the difference between exploration times when both sessions were compared, but it was not able to reverse the difference of the global habituation pattern.

\section{Discussion}

This study was undertaken to test the neuroprotective effects of an ethanolic extract from GP applied simultaneously with resuscitation after ACA. To simulate this clinical relevant situation we used our expensive animal model of ACA (24). This experimental approach is appropriate to study clinical relevant the consequences of ACA in brain $(3,31)$.

We applied an ethanolic extract from GP and used DMSO instead of ethanol as the solvent. This decision was founded on our previous work on brain slices reporting effects of GP on evoked potentials in hippocampus in rats exposed to transient oxygen/glucose deprivation $(15,20)$. In this model it has been demonstrated by others that ethanol caused a dramatic decrease in evoked potentials especially in the hippocampal CA1 region due to modulation of GABA and NMDA receptors $(32,33)$. DMSO doesn't cause such detrimental effects $(34,35)$. With respect to other potential side effects we used the lowest possible DMSO concentration that we estimated to be lower than $0.5 \%$ DMSO within the circulation. At this concentration no side effect was found in vitro (36) and also in vivo (37). Nevertheless, we got a series of DMSO effects which will be discussed in the respective chapters.

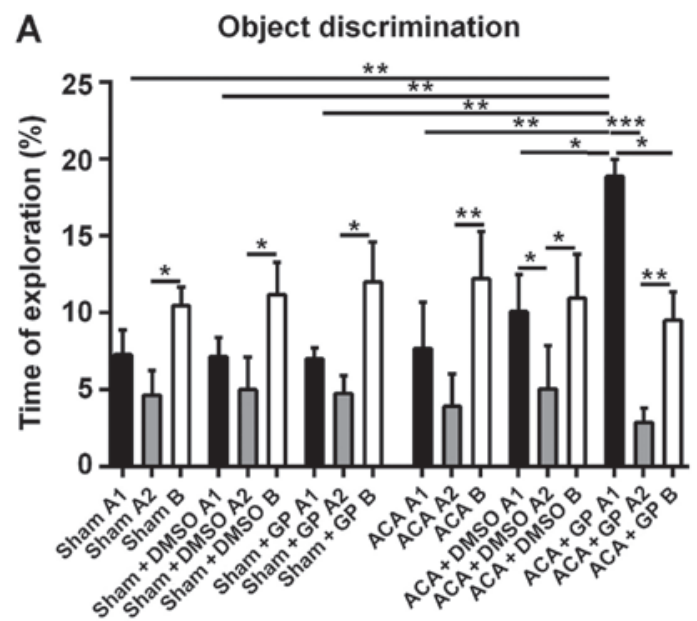

B Global habituation

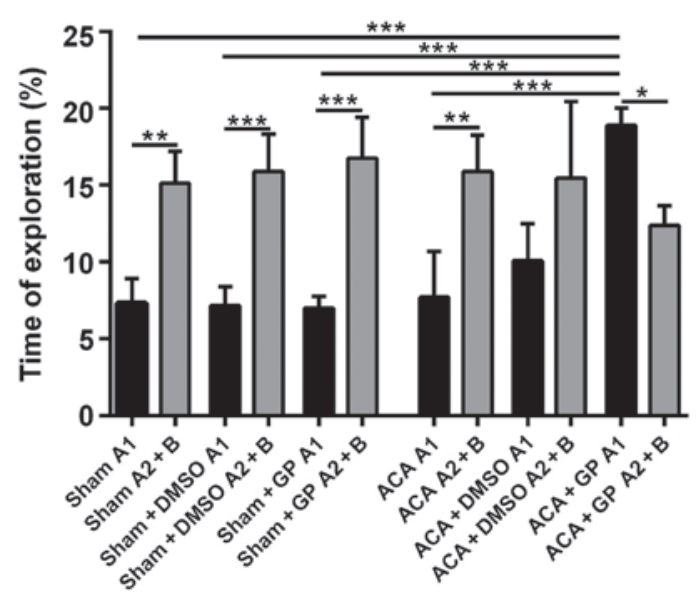

Figure 8. Novel object recognition test 7 days post ACA and resuscitation. (A) Mean of total time (\%) spent to object A1 in the sampling session and to both the familiar object (now A2) and the new object B in the test session. Sham-operated rats explored the novel object B at simultaneous presence of object A (A2) more intensively as the familiar object. This behavioral pattern was unaffected by DMSO or GP. ACA manifested the intensity of object B exploration. The combination of ACA and DMSO led to a significant difference between exploration times of object $\mathrm{A}$ in both sampling and test sessions (A1 vs. A2). GP induced a massive activation of the exploration behavior in the sampling session. The behavioral pattern in the test session was similar to that of ACA-animals without GP-intervention, albeit now both object A2 and object B attracted significant less attention as object A1 in the sampling session. (B) In sham-animals (untreated and DMSO-/GP-treated) and GP-untreated ACA-animals, the totalized exploration time for A2 plus $\mathrm{B}$ in the test trial was significantly higher than for A1 in the sampling trial. DMSO-treatment of ACA-animals reduced this difference; the significance got lost. In GP-treated ACA-animals, this pattern was reversed; the totalized exploration time for A1 in the sampling session was significantly higher than for A2 plus B in the test trail. It was also significantly higher than in all sham-animals and in untreated ACA-animals. Data are presented as the mean $\pm \mathrm{SD}$; Kruskal-Wallis/Dunn's post-hoc test $\left({ }^{*} \mathrm{P}<0.05 ;{ }^{* *} \mathrm{P}<0.005\right.$ $\left.{ }^{* * *} \mathrm{P}<0.001\right)$; all with $\mathrm{n}=5$ /group, ACA, asphyxia cardiac arrest; GP, Gynostemma pentaphyllum; DMSO, dimethyl sulfoxide.

The observed hyperglycaemia during the first 30 min after reperfusion is assumed to result from suppressed insulin secretion and is typical for critical care patients (38). It has been shown that GP stimulates insulin release from islets (39). Since disturbance of glucose homeostasis is a well-known cause of neurodegeneration (reviewed in (40) the prevention of hyperglycaemia by GP should possess neuroprotective potency. A second hallmark of pathophysiological alterations after ACA 
in this period of reperfusion is hypercapnia that has been detected also under our conditions. Hypercapnia contributes to the impairment of brain tissue due to mitochondrial dysfunction (41). In our experiments, GP prevented both post-ischemic hyperglycemia and hypercapnia.

Like GP, DMSO was able to prevent the ACA-mediated increase of $\mathrm{pCO}_{2}$ and blood glucose concentration but in contrast to GP no neuroprotective potency was observed in the presence of DMSO. In contrast to GP, DMSO was shown to suppress insulin secretion from rat pancreatic islet cells (42). Hence, the anti-hyperglycemic effect of DMSO in the ACA animals should be attributed to other mechanisms, e.g., its ability to enhance GLUT4 translocation from intracellular compartments to the plasma membrane. That increases cellular glucose uptake with subsequent reduction of blood glucose levels (43). Together with the ability of DMSO to increase gluconeogenesis a high-glucose-state would be established. High glucose, however, is able to induce extracellular ROS increase and heme oxygenase-1 expression from astrocytes resulting in neuronal apoptosis (44). The neuroprotective potency of GP suggested that GP is able to reverse the effect of its solvent DMSO by its stimulating effect on insulin release (39) and by its capability to attenuate heme oxygenase-1 expression significantly (45).

Reduced hypercapnia by administration of GP and DMSO could be interpreted as a consequence of their anti-hyperglycemic potency which is known to be effective in attenuating post-ischemic hypercapnia (46). Interestingly it was shown that DMSO is able to mimic the neurotoxic effects of hypoxia-hypercapnia, (47) thus counteracting its own anti-hypercapnic/neuroprotective potency. Again, GP seemed to be able to reverse this detrimental DMSO effect. A possible target could be aquaporin 4. Its inhibition, inducible also by gypenosides (48), was helpful in a rat model of hypoxia-hypercapnia-induced brain damage (49).

The hypothesis that GP possesses neuroprotective potency in ACA-dependent brain injury was supported by the histological analysis of the brain. GP, administered once simultaneous with resuscitation, protected the vulnerable hippocampal CA1 region from massive cell injury. Neuroprotection was detected $24 \mathrm{~h}$ after resuscitation but also $7 \mathrm{~d}$ post asphyxia. Thus, the neuroprotective potency of GP turned out to be sustainable. It could be assumed that the observed neuroprotection was caused by an orchestrated action of the major components of the GP extract, Gypenoside LXIII, Gypenoside $\mathrm{Rb}_{3}$ or Gypenoside VIII. The effectiveness of at least Gypenoside VIII [Ginsenoside Rd; $(36,45)$ ] and Gypenoside $\mathrm{Rb}_{3}(50,51)$ against ischemic neurodegeneration is well-documented.

Intact hippocampal structure is required for normal cognitive competences in humans. Therefore, it can be speculated that ACA affects the cognitive potential of patients after transient CA due to neurodegeneration [reviewed by (52)]. This encouraged us to examine attention and recognition skills after ACA by using the novel object recognition test. It is known that under stress conditions hippocampal neurons are challenged by elevated glucocorticoid and glutamate concentrations that are associated with changed cognition abilities $(53,54)$. Thereby, CA1 hippocampal neurons are major targets of corticoids (55), whereby mineralocorticoid and glucocorticoid receptors are co-localized in this cell type (54). Mineralocorticoid receptors are relevant for this stress response by switching from low affinity for corticosterone under normal conditions to high activity at high corticosteroid levels (56). High levels of corticosterone cause increase in exploratory activity of rats (57) paralleled by glutamate-depending increase in working memory. Facilitated working memory has been described when rodents were exposed to acute stress (58). In our experiments the rats failed to develop better performance of object recognition as response to ACA, although ACA should be a massive stressor. Also, the exploration time remained unaffected. Similar results were reported by others (59-61). We would suggest that ACA caused interruption of the physiological response circuit due to degeneration of the respective neurons. When GP was administered the animals responded to ACA with better performance of object recognition and increased exploration time. Obviously, the here demonstrated neuroprotective potency of GP led to an at least partial survival of the physiological response circuits needed for an adequate stress response. Such a positive effect of GP in case of cognition deficits has been described by others too. Zhang et al demonstrated that GP improved cognition impairment induced by chronic cerebral hypoperfusion in rats by suppressing oxidative stress and astrocytic activation $(21,62)$. Hong et al (63) and Joh et al (64) showed that gypenosides are able to ameliorate scopolamine-induced learning deficits in mice. As a possible mechanism they mentioned an increased BDNF expression via the CREB signaling pathway.

Impairment of mitochondria is part of the pathomechanism of ischemia/reperfusion injury including neurodegeneration $(65,66)$. Although neurodegeneration in hippocampus has been observed we did not find a decrease in complex activities of the respiratory chain when evaluated $6 \mathrm{~h}$ after resuscitation. Instead, increased specific activities of complex I + III and complex II + III were detected as a sign of increased energy demand after ACA. From this we conclude that damage of mitochondria could occur earlier with subsequent recovery of mitochondria. This idea fits well with the observed suppression of cell death and stimulation/induction of citrate synthase by GP.

The defense/protection adaption seems to be completed after $24 \mathrm{~h}$ since mitochondrial activities of the respiratory chain complexes widely normalized within this period of time. Initiation of mitochondrial biosynthesis as stress response supports glutamate release and activation of glutamate receptors by providing sufficient ATP for these energy consuming processes (67). Although respiratory chain complex activities normalized within $24 \mathrm{~h}$ we still detected increased CL contents. This particular stress response in hippocampus may contribute to the promotion of mitochondrial ATP synthesis under these conditions. Our data suggest that the vehicle DMSO prevents stimulation of CL synthesis but is not toxic for CL itself. However, a toxic effect of DMSO we observed with respect to complex II + III activity. DMSO had been shown to possess neurotoxic effects similar to ischemia/reperfusion (47). In our experiments, the administration of GP compensated the toxic DMSO effect with respect to complex II + III activity even when solved in DMSO at identical concentration. In line with this finding is the report that GP can stimulate complex I, II and IV (68). Alternatively, GP may directly counteract DMSO effects.

In the presence of GP we found likewise diminished CL contents after ACA treatment. This situation basically differs 
from the effect of DMSO. We found no indication for any toxic GP effect under sham conditions. The combination of ACA and GP administration resulted in elevated citrate synthase activity reflecting stimulation of mitochondrial biogenesis. Under this condition increased demand of ATP can be matched by the higher quantity of mitochondria. It is reasonable to assume that in this situation no stimulation of CL synthesis is required for further increase in mitochondrial ATP generation.

This result emphasized the importance of the early post-resuscitation phase for brain injury and concomitantly for interventional strategies. Because of the structure of the solvent DMSO and gypenosides, it is reasonable to assume that important constituents of the ethanolic GP extract can cross blood brain barrier and cellular membrane systems. The resorption of GP by intestine had been found to operate quickly. GP effects had been observed at least 5 min after i.p. injection. Similar kinetics had been reported by others (69).

We have shown that 6 min of ACA causes early hyperglycaemia and hypercapnia followed by damage of neurons in the hippocampus. This subsequently leads to the inability of cognitive responses in rats. The mechanism of the impairment of neurons includes modifications of mitochondria. Since most of these effects could be attenuated by the administration of GP with resuscitation our results designate the early reperfusion phase as a promising therapeutic window. Moreover, the results underline the need of a careful handling of the popular vehicle DMSO, in some cases even used as control $(70,71)$.

\section{Acknowledgements}

The technical assistance of Susanne Bonifatius, Leona Bück, Stefanie Holze, Daniela Peter and Elke Wölfel Silke Niemann is gratefully acknowledged.

\section{References}

1. Sims NR and Anderson MF: Mitochondrial contributions to tissue damage in stroke. Neurochem Int 40: 511-526, 2002.

2. Schild L and Reiser G: Oxidative stress is involved in the permeabilization of the inner membrane of brain mitochondria exposed to hypoxia/reoxygenation and low micromolar $\mathrm{Ca} 2+$ FEBS J 272: 3593-3601, 2005.

3. Huang L, Applegate PM, Gatling JW, Mangus DB, Zhang J and Applegate RL II: A systematic review of neuroprotective strategies after cardiac arrest: From bench to bedside (part II-comprehensive protection). Med Gas Res 4: 10, 2014.

4. Mangus DB, Huang L, Applegate PM, Gatling JW, Zhang J and Applegate RL II: A systematic review of neuroprotective strategies after cardiac arrest: From bench to bedside (Part I-Protection via specific pathways). Med Gas Res 4: 9, 2014.

5. Sjoberg F and Singer M: The medical use of oxygen: A time for critical reappraisal. J Int Med 274: 505-528, 2013.

6. Dell'anna AM, Scolletta S, Donadello K and Taccone FS: Early neuroprotection after cardiac arrest. Curr Opin Crit Care 20: 250-258, 2014.

7. Llitjos JF, Mira JP, Duranteau J and Cariou A: Hyperoxia toxicity after cardiac arrest: What is the evidence? Ann Intensive Care 6: 23, 2016.

8. Benjamin EJ, Blaha MJ, Chiuve SE, Cushman M, Das SR, Deo R, de Ferranti SD, Floyd J, Fornage M, Gillespie C, et al: Heart disease and stroke statistics-2017 Update: A Report From the American Heart Association. Circulation 135: e146-e603, 2017.

9. Cheng JG: Investigation of the plant jiaogulan and its analogous herb, Wulianmei. Zhong Cao Yao 21: 424, 1990.

10. Mishra RN and Joshi D: Jiao Gu Lan (Gynostemmapentaphyllum): The Chinese Rasayan-Current Research Scenario. Int J Res Pharm Biom Sci 2: 1483-1502, 2011.
11. Chen J: Antistress action of Gynostemma pentaphyllum. Chinese Tradit Patent Med 11: 31-32, 1989.

12. Shang L, Liu J, Zhu Q, Zhao L, Feng Y, Wang X, Cao W and $\mathrm{Xin} \mathrm{H}$ : Gypenosides protect primary cultures of rat cortical cells against oxidative neurotoxicity. Brain Res 1102: 163-174, 2006.

13. Norberg A, Hoa NK, Liepinsh E, Van Phan D, Thuan ND, Jörnvall H, Sillard R and Ostenson CG: A novel insulin-releasing substance, phanoside, from the plant Gynostemma pentaphyllum. J Biol Chem 279: 41361-41367, 2004.

14. Wang P, Niu L, Gao L, Li WX, Jia D, Wang XL and Gao GD: Neuroprotective effect of gypenosides against oxidative injury in the substantia nigra of a mouse model of Parkinson's disease. J Int Med Res 38: 1084-1092, 2010.

15. Schild L, Roth A, Keilhoff G, Gardemann A and Brödemann R: Protection of hippocampal slices against hypoxia/hypoglycemia injury by a Gynostemma pentaphyllum extract. Phytomedicine 16: 734-743, 2009.

16. Paradies G, Paradies V, Ruggiero FM and Petrosillo G: Cardiolipin and mitochondrial function in health and disease. Antioxid Redox Signal 20: 1925-1953, 2014.

17. Mileykovskaya E and Dowhan W: Cardiolipin-dependent formation of mitochondrial respiratory supercomplexes. Chem Phys Lipids 179: 42-48, 2014.

18. Nakahara I, Kikuchi H, Taki W, Nishi S, Kito M, Yonekawa Y, Goto $\mathrm{Y}$ and Ogata N: Changes in major phospholipids of mitochondria during postischemic reperfusion in rat brain. J Neurosurg 76: 244-250, 1992.

19. Ji J, Baart S, Vikulina AS, Clark RS, Anthonymuthu TS, Tyurin VA, Du L, St Croix CM, Tyurina YY, Lewis J, et al: Deciphering of mitochondrial cardiolipin oxidative signaling in cerebral ischemia-reperfusion. J Cereb Blood Flow Metab 35: 319-328, 2015.

20. Schild L, Cotte T, Keilhoff G and Brodemann R: Preconditioning of brain slices against hypoxia induced injury by a Gynostemma pentaphyllum extract-stimulation of anti-oxidative enzyme expression. Phytomedicine 19: 812-818, 2012.

21. Zhang GL, Deng JP, Wang BH, Zhao ZW, Li J, Gao L, Liu BL, Xong JR, Guo XD, Yan ZQ and Gao GD: Gypenosides improve cognitive impairment induced by chronic cerebral hypoperfusion in rats by suppressing oxidative stress and astrocytic activation. Behav Pharmacol 22: 633-644, 2011.

22. Keilhoff G, John R, Langnaese K, Schweizer $H$ and Ebmeyer U: Triggered by asphyxia neurogenesis seems not to be an endogenous repair mechanism, gliogenesis more like it. Neuroscience 171: 869-884, 2010.

23. Keilhoff G, Schweizer H, John R, Langnaese K and Ebmeyer U: Minocycline neuroprotection in a rat model of asphyxial cardiac arrest is limited. Resuscitation 82: 341-349, 2011.

24. Keilhoff G, Titze M, Esser T, Langnaese K and Ebmeyer U: Constitutive and functional expression of YB-1 in microglial cells. Neuroscience 301: 439-453, 2015.

25. Schild L, Chen BH, Makarov P, Kattengell K, Heinitz K and Keilhoff G: Selective induction of apoptosis in glioma tumour cells by a Gynostemma pentaphyllum extract. Phytomedicine 17: 589-597, 2010.

26. Kao TH, Huang SC, Inbaraj BS and Chen BH: Determination of flavonoids and saponins in Gynostemma pentaphyllum (Thunb.) Makino by liquid chromatography-mass spectrometry. Anal Chim Acta 626: 200-211, 2008.

27. Moellering $\mathrm{H}$ and Gruber W: Determination of citrate with citrate lyase. Anal Biochem 17: 369-376, 1966.

28. Martens JC, Keilhoff G, Halangk W, Wartmann T, Gardemann A, Päge I and Schild L: Lipidomic analysis of molecular cardiolipin species in livers exposed to ischemia/reperfusion. Mol Cell Biochem 400: 253-263, 2015.

29. Bradford MM: A rapid and sensitive method for the quantitation of microgram quantities of protein utilizing the principle of protein-dye binding. Anal Biochem 72: 248-254, 1976.

30. Ennaceur A and Delacour J: A new one-trial test for neurobiological studies of memory in rats. 1: Behavioral data. Behav Brain Res 31: 47-59, 1988.

31. Kandiah P, Ortega S and Torbey MT: Biomarkers and neuroimaging of brain injury after cardiac arrest. Semin Neurol 26: 413-421, 2006.

32. Schummers $\mathrm{J}$ and Browning MD: Evidence for a role for GABA(A) and NMDA receptors in ethanol inhibition of long-term potentiation. Brain Res Mol Brain Res 94: 9-14, 2001.

33. Steffensen SC, Nie Z, Criado JR and Siggins GR: Ethanol inhibition of $\mathrm{N}$-methyl-D-aspartate responses involves presynaptic gamma-aminobutyric acid(B) receptors. J Pharmacol Exp Ther 294: 637-647, 2000. 
34. Albertson TE and Joy RM: Increased inhibition in dentate gyrus granule cells following exposure to GABA-uptake blockers. Brain Res 435: 283-292, 1987.

35. Laaris N, Good CH and Lupica CR: Delta9-tetrahydrocannabinol is a full agonist at CB1 receptors on GABA neuron axon terminals in the hippocampus. Neuropharmacology 59: 121-127, 2010

36. Zhang G, Xia F, Zhang Y, Zhang X, Cao Y, Wang L, Liu X, Zhao $\mathrm{G}$ and Shi M: Ginsenoside Rd is efficacious against acute ischemic stroke by suppressing microglial proteasome-mediated inflammation. Mol Neurobiol 53: 2529-2540, 2016.

37. Bakar B, Kose EA, Sonal S, Alhan A, Kilinc K and Keskil IS Evaluation of the neurotoxicity of DMSO infused into the carotid artery of rat. Injury 43: 315-322, 2012

38. Nurmi J, Boyd J, Anttalainen N, Westerbacka J and Kuisma M: Early increase in blood glucose in patients resuscitated from out-of-hospital ventricular fibrillation predicts poor outcome. Diabetes Care 35: 510-512, 2012.

39. Lokman EF, Gu HF, Wan Mohamud WN and östenson CG Evaluation of antidiabetic effects of the traditional medicinal plant gynostemma pentaphyllum and the possible mechanisms of insulin release. Evid Based Complement Alternat Med 2015: $120572,2015$.

40. Hassan M, Sehgal SA and Rashid S: Regulatory cascade of neuronal loss and glucose metabolism. CNS Neurol Disord Drug Targets 13: 1232-1245, 2014

41. Douglas RM, Ryu J, Kanaan A, Del Carmen Rivero M, Dugan LL, Haddad GG and Ali SS: Neuronal death during combined intermittent hypoxia/hypercapnia is due to mitochondrial dysfunction. Am J Physiol Cell Physiol 298: C1594-C1602, 2010.

42. Sakonju I, Taura Y, Inayoshi Y, Suzuki T, Takimoto K Nakaichi $M$ and Nakama S: Cryopreservation of isolated rat islets of Langerhans in the presence of ethylene glycol or dimethyl sulfoxide: Evaluation of toxicity and the dynamic pattern of subsequent insulin release in vitro. Cryobiology 33 354-362, 1996.

43. Berenguer M, Zhang J, Bruce MC, Martinez L, Gonzalez T, Gurtovenko AA, Xu T, Le Marchand-Brustel Y and Govers R: Dimethyl sulfoxide enhances GLUT4 translocation through a reduction in GLUT4 endocytosis in insulin-stimulated 3T3-L1 adipocytes. Biochimie 93: 697-709, 2011.

44. Yang CM, Lin CC and Hsieh HL: High-glucose-derived oxidative stress-dependent heme oxygenase-1 expression from astrocytes contributes to the neuronal apoptosis. Mol Neurobiol 54: 470-483, 2017.

45. Ye R, Zhao G and Liu X: Ginsenoside Rd for acute ischemic stroke: translating from bench to bedside. Expert Rev Neurother 13: 603-613, 2013.

46. Kim YB, Gidday JM, Gonzales ER, Shah AR and Park TS Effect of hypoglycemia on postischemic cortical blood flow, hypercapnic reactivity and interstitial adenosine concentration. J Neurosurg 81: 877-884, 1994

47. Yu LS, Fan YY, Ye G, Li J, Feng XP, Lin K, Dong M and Wang Z: Curcumin alleviates brain edema by lowering AQP4 expression levels in a rat model of hypoxia-hypercapnia-induced brain damage. Exp Ther Med 11: 709-716, 2016.

48. Zhou Y, Li HQ, Lu L, Fu DL, Liu AJ, Li JH and Zheng GQ: Ginsenoside Rg1 provides neuroprotection against blood brain barrier disruption and neurological injury in a rat model of cerebral ischemia/reperfusion through downregulation of aquaporin 4 expression. Phytomedicine 21: 998-1003, 2014.

49. Yu H, Qi GL, Wang J, Chen L, Deng Z, Zhao YS, Lei SS and Zhu XQ: Aquaporin 4 inhibition decreased synthesis of cytokines by acetazolamide in the hippocampus of rats with pentrazol-induced chronic epilepsy. Genet Mol Res: 15, 2016.

50. Jiang $S$, Miao $B$, Song $X$ and Jiang $Z$ : Inactivation of $\mathrm{GABA}(\mathrm{A})$ receptor reduces ginsenoside $\mathrm{Rb} 3$ neuroprotection in mouse hippocampal slices after oxygen-glucose deprivation. J Ethnopharmacol 133: 914-916, 2011

51. Zhu JR, Tao YF, Lou S and Wu ZM: Protective effects of ginsenoside $\mathrm{Rb}(3)$ on oxygen and glucose deprivation-induced ischemic injury in PC12 cells. Acta Pharmacol Sin 31: 273-280, 2010.
52. Green CR, Botha JA and Tiruvoipati R: Cognitive function, quality of life and mental health in survivors of our-of-hospital cardiac arrest: A review. Anaesth Intensive Care 43: 568-576, 2015.

53. Popoli M, Yan Z, McEwen BS and Sanacora G: The stressed synapse: The impact of stress and glucocorticoids on glutamate transmission. Nat Rev Neurosci 13: 22-37, 2012.

54. Takeda A and Tamano H: Proposed glucocorticoid-mediated zinc signaling in the hippocampus. Metallomics 4: 614-618, 2012.

55. Joels M: Functional actions of corticosteroids in the hippocampus. Eur J Pharmacol 583: 312-321, 2008.

56. de Kloet ER, Karst H and Joels M: Corticosteroid hormones in the central stress response: Quick-and-slow. Front Neuroendocrinol 29: 268-272, 2008.

57. Sutt S, Raud S, Abramov U, Innos J, Luuk H, Plaas M, Kõks S, Zilmer K, Mahlapuu R, Zilmer M and Vasar E: Relation of exploratory behaviour to plasma corticosterone and Wfs1 gene expression in Wistar rats. J Psychopharmacol 24: 905-913, 2010.

58. Yuen EY, Liu W, Karatsoreos IN, Ren Y, Feng J, McEwen BS and Yan Z: Mechanisms for acute stress-induced enhancement of glutamatergic transmission and working memory. Mol Psychiatry 16: 156-170, 2011.

59. Hoge $\mathrm{J}$ and Kesner RP: Role of CA3 and CA1 subregions of the dorsal hippocampus on temporal processing of objects. Neurobiol Learn Mem 88: 225-231, 2007.

60. Vago DR, Bevan A and Kesner RP: The role of the direct perforant path input to the CA1 subregion of the dorsal hippocampus in memory retention and retrieval. Hippocampus 17: 977-987, 2007.

61. Piterkin P, Cole E, Cossette MP, Gaskin S and Mumby DG: A limited role for the hippocampus in the modulation of novel-object preference by contextual cues. Leam Mem 15: 785-791, 2008.

62. Zhang G, Zhao Z, Gao L, Deng J, Wang B, Xu D, Liu B, Qu Y, Yu J, Li J and Gao G: Gypenoside attenuates white matter lesions induced by chronic cerebral hypoperfusion in rats. Pharmacol Biochem Behav 99: 42-51, 2011.

63. Hong SW, Yang JH, Joh EH, Kim HJ and Kim DH: Gypenoside TN-2 ameliorates scopolamine-induced learning deficit in mice. J Ethnopharmacol 134: 1010-1013, 2011.

64. Joh EH, Yang JW and Kim DH: Gypenoside LXXIV ameliorates scopolamine-induced learning deficit in mice. Planta Med 76: 793-795, 2010

65. Hou ST and MacManus JP: Molecular mechanisms of cerebral ischemia-induced neuronal death. Int Rev Cytol 221: 93-148, 2002.

66. Szeto HH: Mitochondria-targeted cytoprotective peptides for ischemia-reperfusion injury. Antioxid Redox Signal 10: 601-619, 2008.

67. Osborne DM, Pearson-Leary J and McNay EC: The neuroenergetics of stress hormones in the hippocampus and implications for memory. Front Neurosci 9: 164, 2015.

68. Yu H, Guan Q, Guo L, Zhang H, Pang X, Cheng Y, Zhang X and Sun Y: Gypenosides alleviate myocardial ischemia-reperfusion injury via attenuation of oxidative stress and preservation of mitochondrial function in rat heart. Cell Stress Chaperones 21: 429-437, 2016.

69. Chen DJ, Hu HG, Xing SF, Gao YJ, Xu SF and Piao XL: Metabolic profiling of Gynostemma pentaphyllum extract in rat serum, urine and faeces after oral administration. J Chromatogr B Analyt Technol Biomed Life Sci 969: 42-52, 2014.

70. Nejad KH, Gharib-Naseri MK, Sarkaki A, Dianat M, Badavi M and Farbood Y: Effects of ellagic acid pretreatment on renal functions disturbances induced by global cerebral ischemic-reperfusion in rat. Iran J Basic Med Sci 20: 75-82, 2017.

71. Filippone SM, Samidurai A, Roh SK, Cain CK, He J, Salloum FN, Kukreja RC and Das A: Reperfusion Therapy with Rapamycin Attenuates Myocardial Infarction through Activation of AKT and ERK. Oxid Med Cell Longev 2017: 4619720, 2017. 